\title{
The Pharmacological Effects of Triterpenoids from Ganoderma lucidum and the Regulation of Its Biosynthesis
}

\author{
Siye Chen \\ Molecular Plant Breeding and Pathology Cluster, Plant Science Group, Wageningen University and Research, Wageningen \\ 6708PB, The Netherlands \\ Email: siye_chen@foxmail.com
}

How to cite this paper: Chen, S.Y. (2020) The Pharmacological Effects of Triterpenoids from Ganoderma lucidum and the Regulation of Its Biosynthesis. Advances in Biological Chemistry, 10, 55-65. https://doi.org/10.4236/abc.2020.102005

Received: March 18, 2020

Accepted: April 20, 2020

Published: April 23, 2020

Copyright $\odot 2020$ by author(s) and Scientific Research Publishing Inc. This work is licensed under the Creative Commons Attribution International License (CC BY 4.0).

http://creativecommons.org/licenses/by/4.0/

\section{(c) (i) Open Access}

\begin{abstract}
Ganoderma lucidum is a wildly used medicinal mushroom and has been used in East Asia because of its health-benefit effects. G. lucidum contains various compounds with different biological activities, which include anti-tumour, anti-viral, and anti-malaria effect. Ganoderic acid (GA) is a triterpenoid from G. lucidum. The biosynthesis of GA in G. lucidum is induced by stressors including various elicitors or signaling molecules, and artificially placing elicitors would enhance GA production. In this paper, bioactivities and potential applications of GAs would be focused, and the elicitation strategies for GA production are also highlighted based on the fundamental role of ROS, JA, $\mathrm{Ca}^{2+}$ and NO, which would shed light to developing a novel approach to manipulating the biosynthesis of GA in the future.
\end{abstract}

\section{Keywords}

Ganoderma lucidum, Ganoderic Acids, Biosynthesis, Pharmacological Effects

\section{Introduction}

Ganoderma lucidum, commonly known as Lingzhi in Chinese, is a woody basidiomycetes mushroom belonging to the family of Ganodermaceae [1]. G. lucidum has been used for two millennia as herbal medicine mainly in Asian areas. It had the efficacy of improving immunity, soothing the nerves, protecting the liver, detoxification and prolonging life expectancy [2]. Over 400 bioactive compounds were found in $G$. lucidum such as polysaccharides and triterpenoids. The most important bioactive compounds in G. lucidum are triterpenoids which are known as ganoderic acids (GAs). The medicinal effects of GA were verified, 
which include anti-tumor, anti-malaria, anti-microbial, anti-inflammatory, and anti-viral effects [2] [3] [4] [5] [6]. Due to the commercial and pharmacological value of GA, biosynthesis of GA by cell factories received more and more attention in recent years [7]. However, the productivity and yield of GA by cell culture technology are still very low in spite of many years of efforts [8].

Elicitors are extrinsic molecules often associated with plant pests and pathogen [9]. Generally, elicitors are regarded as a signal molecule and could be recognized by the receptor on the surface of the plant plasma membrane or endomembrane [10]. Subsequently, recognition events would induce a signal-transduction cascade leading to activate biosynthesis of transcription factors [11]. Those transcription factors regulate the expression of the genes which are involved in the biosynthesis of secondary metabolisms, such as phytoalexins, flavones, triterpenoid and other defense-related compounds [9]. An obvious positive correlation between elicitor addition and GA accumulation has been reported in the previous study [11]. Besides, these elicitors have been well studied in G. lucidum within the context of mycelial cultures [12]. So that elicitor would be considered as the main manner to enhance GA production in the future [13].

\section{Pharmacological Effects of GAs}

Hajjaj, Macé [14] reported that the pharmacological effects of GAs are mainly related to the hydroxylation of their structure. For example, the ganoderic acid $\mathrm{A}$ (GA-A) is hydroxylated at C-3, while ganoderic acid D (GA-D) is hydroxylated at positions 7 and 15 [15] [16]. Other triterpenes with acetoxy or hydroxyl groups at locations 3,7 or 15 , such as ganoderic acid C (GA-C), ganoderic acid $B$ (GA-B), ganoderic acid $\mathrm{Me}$ (GA-Me) ganoderic acid $\mathrm{K}$ (GA-K) were also proven as Bioactivities metabolism [17] [18] [19]. The recent research on bioactivities of Ganoderma are showed in Table 1 and summarized as follows.

Table 1. Bioactivities of Ganoderma metabolites.

\begin{tabular}{ccc}
\hline Bioactivity & Active metabolite & Reference \\
\hline \multirow{2}{*}{ Anti-tumor } & Ganoderic acid S & Liu et al. (2009) \\
& Ganoderic acid B & Hsu et al. (2008) \\
& Ganoderic acid F & Gao et al. (2006) \\
Ganoderic acid Y & Jiang et al. (2011) \\
Anti-HIV & Ganoderic acid B & Kang et al. (2015) \\
Anti-malaria & Ganoderic acid beta & Paydary et al. (2013) \\
& Ganoderic acid F & Lakornwong et al. (2014) \\
Anti-hypertensive & Schisanlactone B & Lakornwong et al. (2014) \\
Anti-hepatotoxic & Ganoderiol F & Dondorp et al. (2009) \\
Hemostasis & Ganoderic acid K & Kabir et al. (1988) \\
\hline
\end{tabular}




\subsection{Antitumor Activity}

Ganoderic acids are well acknowledged as bioactive metabolites to improve the function of the human immune system [20]. Toth, Bang [21] firstly reported antitumor activity of GA, which is triggering apoptosis in cervical carcinoma cell in vitro. Yue, Cao [16] found GA would inhibit the proliferation of HeLa human cervical carcinoma cells by keeping cell cycle arrest at the G2 phase. Furthermore, the anti-metastasis effects of GA-Me were reported [22]. GA-Me could inhibit cancer cell metastasis by increasing of cell adhesion and decreasing of cell motility, and it also suppresses the expression of MMP2 gene which was involved in the progression and metastasis of many forms of cancer [23]. Therefore, GA-Me could be considered as a promising anti-metastatic compound [24]. Also, there are many reports on the antitumor activity of another ganoderic acid. Hsu, Yu [17] discovered that GA-B could inhibit the growth of some tumours cell lines and trigger apoptosis in human leukemia HL-60 cells. Gao, Hirakawa [19] showed the in vivo antitumor effects of the ganoderic acid F (GA-F). This is demonstrated by cytotoxicity assay in lung carcinoma cell (LLC)-bearing mice. GA-F showed a remarkable activity in cytotoxicity and strongly inhibited the growth of the tumour without obvious side effects. Jiang, Jedinak [25] reported that GA-Y would block the expression of the cell cycle regulatory protein CDC20, thereby proliferation and development of invasive and metastatic human breast cancer cells was also inhibited.

\subsection{Anti-HIV Activity}

Acquired immunodeficiency syndrome (AIDS), caused by HIV, is highly contagious and affect millions of people all over the world. Postponing the development of AIDS is the main goals of treatment approaches for HIV [26]. Several articles reported that ganoderic acids could inhibit the progression of HIV including ganoderic acid beta, GA-A, and GA-B. Those ganoderic acids have been shown to have significant anti-human immunodeficiency virus protease activity, with half maximal inhibitory concentration values of 20 - 90 Millimole per millilitre [27]. In one of the earlier research, El-Mekkawy, Meselhy [28] successfully isolated fifteen compounds from G. lucidum including ganoderic acid. They found that those compounds had strong inhibitory activity against HIV-1 proteases. Recently, Kang, Mutakin [29] also indicated that ganoderic acid B possessed the highest inhibiting activity to HIV-protease of four tested triterpenoids. In addition, Zhang, Ip [30] reported the extractive of G. lucidum could inhibit HIV-1 reverse transcriptase. The researches mentioned above suggest the huge potential of ganoderic acid for HIV treatment.

\subsection{Anti-Malaria Activity}

According to the estimation of Centers for Diseases Control and Prevention, there are 429,000 people died from malaria infection worldwide in 2015 [31]. Malaria is a disease caused by a Plasmodium, and it can be treated by artemisi- 
nin. However, Dondorp, Nosten [32] reported the artemisinin resistance in Plasmodium falciparum. Lakornwong, Kanokmedhakul [33] found the anti-malaria activity of triterpene isolated from Ganoderma. They cultured Ganoderma sp. KM01 and isolated eleven different from the mycelium, and then the antimalarial activity against $P$. falciparum of those triterpene was investigated. In vitro, $P$. falciparum assay illustrated that GA-F and schisanlactone $B$ caused half $P$. falciparum death with the dose ranging from 6.0 to $10.0 \mu \mathrm{mol} / \mathrm{L}$. Except for triterpene, based on computational molecular docking, GA-F and ganoderiol $F$ also have the potential to restrict the growth of $P$. falciparum by inhibiting aspartic protease [33]. Secondary metabolites such as triterpene produced by Ganoderma seem to have the potential to inhibit malaria but still require more studies.

\subsection{Other Pharmacological Effects of GAs}

Since the last century, the pharmacological effects of ganoderic acids were already well studied. Hirotani, Ino [34] reported that GA-B, GA-D, GA-F, and GA-K have antihypertensive effects by inhibiting the activity of angiotensin-converting enzymes especially GA-K, which had the highest inhibitory effect with a half maximal inhibitory concentration of $4.7 \mu \mathrm{mol} / \mathrm{L}$. Kabir, Kimura [35] also found the GA effects on reducing blood pressure and lipid levels in hypertensive rats. Furthermore, the antihepatotoxic activity of ganoderic acid $\mathrm{R}$ and ganoderic acid S, which was isolated from the cultured mycelia of G. lucidum, was proved in the galactosamine-induced cytotoxic test with primary rat hepatocytes [34]. Besides, Wang, Chen [36] reported that ganodermic acid S could induce the aggregation of human platelet. At a concentration of $20 \mu \mathrm{mol} / \mathrm{L}$, the existing of ganodermic acid $S$ caused platelet aggregation. Above the threshold, the extents of cell aggregation showed a linear relationship with agent concentration.

\section{The Biosynthetic Pathway of GAs}

The pathway of GA biosynthesis is not fully understood yet. Earlier biogenetic research illustrates that GA is biosynthesised via the mevalonate-isoprenoid pathway from glucose until lanosterol in G. lucidum [37] [38] [39] [40] [41].

Firstly, the biosynthesis of 3-hydroxy-3-methylglutaryl-CoA (HMG-CoA) catalyzed by 3-hydroxy-3-methylglutaric acid coenzyme. Then HMG-CoA is converted to mevalonate (MVA) to and further to isopentenyl-pyrophosphate (IPP). Squalene synthase (SQS) catalyse the enzymatic step toward squalene biosynthesis [42]. Finally, lanosterol is formed from squalene under the catalysis of oxidosqualene cyclase (OSC) (Figure 1). Lanosterol formed by the cyclization of 2,3-oxidosqualene is the ring skeleton of GAs. This cyclization is catalysed by lanostane synthase (LS). However, further steps from lanosterol to GA, which may include oxidation, reduction, and acylation reactions are yet unclear [43].

In addition, it was proposed that a series of oxidation-reduction steps are 


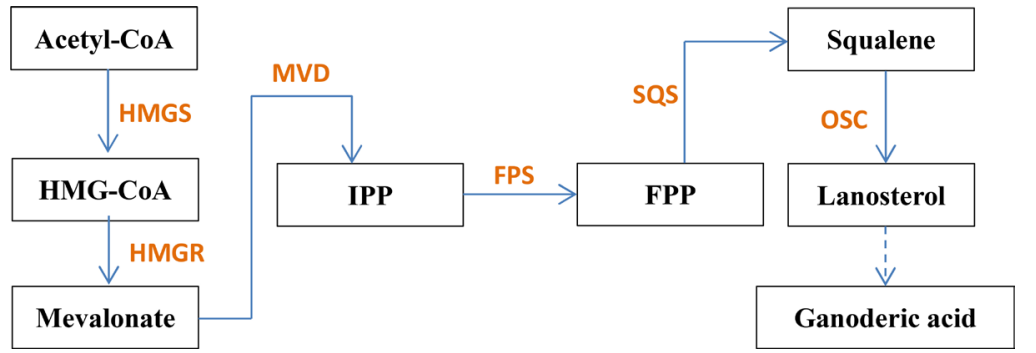

Figure 1. Biosynthetic pathway of GA. Dashed lines indicates this step is yet unclear. HMG-CoA, hydroxy-3-methylglutaryl-Coenzyme A; HMGS, HMG-CoA synthase; HMGR, HMG-CoA reductase; MVD, mevalonate-5-pyrophosphate decarboxylase; IPP, isopentenyl-pyrophosphate; FPS, farnesyl pyrophosphate synthase; FPP, farnesyl diphosphate; SQS, squalene synthase; OSC, oxidosqualene cyclase.

likely involved in the post-lanosterol modifications [44]. The genome analysis of G. lucidum reveals cytochromes $\mathrm{P} 450$ (CYPs), including $24 \mathrm{P} 450$ clusters and 78 P450 genes might involve in catalysing the lanosterol skeletons into triterpenoids [45]. Even though the pathway of GA biosynthesis is not fully understood, the knowledge of the genes and enzymes involved in the GA biosynthetic pathway should lead to an in-depth understanding of the regulation and physiology of GA biosynthesis.

\section{Elicitor for Regulating of Ganoderic Acid Biosynthesis}

\subsection{Reactive Oxygen Species Inducer}

Reactive oxygen species (ROS) is a by-product of biological aerobic metabolism, including superoxide anion, hydrogen peroxide and singlet oxygen. Zhang and Zhong [46] reported the higher oxygen concentration would increase individual GAs production, and more spores and higher total GA content were obtained at an oxygen level of $80 \%$. Feng, Zhang [47] found oleic acid has an effect on promoting in GA accumulation because the oleic acid addition improved the level of dissolved oxygen in liquid submerged fermentation of G. lucidum. Aspirin is known as an agent which could induce oxidative stress by causing mitochondrial dysfunction. When the G. lucidum was cultured with $2 \mathrm{mmol} / \mathrm{L}$ aspirin, ROS production was enhanced in mycelia. And ROS was further accumulated with the aspirin concentration increasing to $4 \mathrm{mmol} / \mathrm{L}$. You, Lee [48] found that GA production has increased fourfold in the submerged fermentation containing 4 $\mathrm{mmol} / \mathrm{L}$ aspirin. These results illustrated that ROS contribute to the regulation and production of GA and biosynthesis in G. lucidum.

\subsection{Metal Ions}

Metal ions such as calcium ion and iron ion play an essential role in the biosynthesis of metabolism in various organisms. The application of $\mathrm{Ca}^{2+}$ is helpful for GA biosynthesis. Xu and Zhong [49] reported the induction of $\mathrm{Ca}^{2+}$ result in a threefold growth in the production of total GAs, accompanied by higher expression of three biosynthetic genes $L S, H M G R$ and $S Q S$. Moreover, the binding 
sites of two transcription factors ( $C R Z 1$ and $A r e A)$ which are involved in nitrogen regulation both were found in the promoter regions of $L S, H M G R$ and $S Q S$. This phenomenon means that the biosynthesis of GAs may be regulated by the concentration of nitrogen and calcium ion synergistically [50]. Therefore, a new integrated strategy was developed, which is adding $\mathrm{Ca}^{2+}$ and reducing nitrogen simultaneously to promote GA biosynthesis. Applying this strategy up-regulated transcriptional level of the biosynthetic gene ( $L S, H M G R$ and $S Q S$ ) and increase the supplies of precursors, resulting in improve the production of GA by nine-fold compared with controls. In addition, the integrated strategy appeared to synergistic activity. The maximum output of GA production regulated by the integrated strategy was $1.87 \mathrm{~g} / 100 \mathrm{~g}$ in dry cell weight, which was nearly fivefold higher than the GA production regulated by either adding $\mathrm{Ca}^{2+}$ or reducing nitrogen [51].

\subsection{Phytohormones}

Plant hormones have been studied as elicitor for many years. The most studied one is Jasmonic acid (JA) and its derivatives such as methyl jasmonate (MeJA). They are responsible for signal transduction processes which regulate the expression of defence genes in plants [52]. In the liquid culture of G. lucidum mycelium, artificially adding MeJA could induce rising of the content of endogenous MeJA. Introduction of $254 \mu \mathrm{M}$ MeJA would improve GA content by $45.3 \%$ and up-regulated the transcriptional level of GA biosynthetic genes, such as $M V D, H M G S$ and $O S C$ [53]. Ethylene (ET) is a hydrocarbon, which is involved in biochemical and morphological changes during the process of fruit ripening and senescing [54]. In CYM medium, applying of $15 \mathrm{mM}$ ethephon could enhance the growth of $G$. lucidum mycelia, resulting in GA production went up by 90\%. Besides, the transcription levels of $O S C$ and $H M G R$ were up-regulated by threefold and fourfold compared to the control group [55]. Salicylic acid (SA), a type of phenolic acid with an essential role in plant defence systems, is admitted to inducing systemically acquired resistance to many pathogens [52]. The addition of SA resulted in more GA accumulation by enhancing the gene expression of $H M G R$ and $L S$ in the mycelium of G. lucidum [56].

There are already many reports focusing on the crosstalk among ET, JA and SA signalling pathways, but the knowledge about crosstalk among these plant hormones in G. lucidum is very limited [57]. Therefore, more research about hormonal crosstalk in G. lucidum might open up perspectives to manipulate the biosynthesis of GA.

\subsection{Nitric Oxide}

Nitric oxide (NO) is a gaseous signaling molecule [58]. It is a key bioactive molecule, playing a role in many fungi physiological processes such as spore germination, hyphal growth, and the responses to environmental stresses [59]. In $G$. oregonense, heat tolerance could be enhanced by NO through increasing the ex- 
pression of $H S P$ genes such as HSP30, HSP70, and HSP104 [60]. Introduction of the NO donor such as sodium nitroprusside (SNP) would increase GA production [61]. In submerged fermented mycelia of G. lucidum, the introduction of the SNP at the concentration of $5 \mathrm{mmol} / \mathrm{L}$ could improve GA content by $40.94 \%$. After 72 hours of introducing SNP, the expression of GA biosynthetic genes including $A C A T$ and $S E$ was detected to be up-regulated.

\section{Conclusions and Prospects}

Over the centuries, G. lucidum has been extensively used as a medical mushroom. It produces huge amounts of bioactive secondary metabolites such as GA from its mycelia or fruiting body. From the perspective of pharmacology, those secondary metabolites have many biological activities, which include antitumor, antimicrobial, anti-malaria, antihepatotoxic and blood pressure reduction activity. From the aspect from fungi, the main function of secondary metabolites is adaptive responses to biotic or abiotic stresses. Application of elicitors into in vitro cultures would mimic the biotic or abiotic attacks, resulting in the biosynthesis of secondary metabolites. Introduction of elicitors such as heavy metal ion, phytohormones, hydrogen peroxide, aspirin, and NO into cultured mycelia of in G. lucidum have been recognized as effective ways to promote GA accumulation. Furthermore, other approaches such as genetic modification and nutrition regulation can be integrated with elicitation strategy to get a more dramatic improvement of GA productivity in G. lucidum. However, elicitors induce the accumulation of secondary metabolite, usually accompany with blocked cell growth, thereby lower total GA production. Therefore, dissecting the response networks of G. lucidum to various elicitor molecules is important for developing novel strategies for enhancing GA production.

As a whole, further research about the signal transduction induced by elicitor in G. lucidum will benefit in improving the yields of specific secondary metabolites. Moreover, it would also further promote the efficiency and accuracy of genetic modification by transcription factors. According to the information from metabolomics studies, manipulating metabolic pathways in G. lucidum with different biotechnology could offer insight for higher productivity of GAs.

\section{Conflicts of Interest}

The author declares no conflicts of interest regarding the publication of this paper.

\section{References}

[1] Babu, P.D. and Subhasree, R. (2008) The Sacred Mushroom "Reishi": A Review. The American-Eurasian Journal of Botany, 1, 107-110.

[2] Joseph, S., et al. (2009) Antioxidative and Antiinflammatory Activities of the Chloroform Extract of Ganoderma lucidum Found in South India. Scientia Pharmaceutica, 77, 111-122. https://doi.org/10.3797/scipharm.0808-17

[3] Kao, C., et al. (2013) Anti-Cancer Activities of Ganoderma lucidum: Active Ingre- 
dients and Pathways. Functional Foods in Health and Disease, 3, 48-65. https://doi.org/10.31989/ffhd.v3i2.65

[4] Batra, P., Sharma, A.K. and Khajuria, R. (2013) Probing Lingzhi or Reishi Medicinal Mushroom Ganoderma lucidum (Higher Basidiomycetes): A Bitter Mushroom with Amazing Health Benefits. International Journal of Medicinal Mushrooms, 15, 127-143. https://doi.org/10.1615/Int/MedMushr.v15.i2.20

[5] Liao, S.-F., et al. (2013) Immunization of Fucose-Containing Polysaccharides from Reishi Mushroom Induces Antibodies to Tumor-Associated Globo H-Series Epitopes. Proceedings of the National Academy of Sciences, 110, 13809-13814. https://doi.org/10.1073/pnas.1312457110

[6] Chen, W., et al. (2005) Effects of Ganoderma lucidum Polysaccharides on Serum Lipids and Lipoperoxidation in Experimental Hyperlipidemic Rats. China Journal of Chinese Materia Medica, 30, 1358-1360.

[7] Shi, L., et al. (2012) Development of a Simple and Efficient Transformation System for the Basidiomycetous Medicinal Fungus Ganoderma lucidum. World Journal of Microbiology and Biotechnology, 28, 283-291. https://doi.org/10.1007/s11274-011-0818-Z

[8] Tang, Y.J. and Zhong, J.J. (2003) Scale-Up of a Liquid Static Culture Process for Hyperproduction of Ganoderic Acid by the Medicinal Mushroom Ganodermalucidum. Biotechnology Progress, 19, 1842-1846. https://doi.org/10.1021/bp0341592

[9] Mishra, A.K., Sharma, K. and Misra, R.S. (2012) Elicitor Recognition, Signal Transduction and Induced Resistance in Plants. Journal of Plant Interactions, 7, 95-120. https://doi.org/10.1080/17429145.2011.597517

[10] Zhao, J., Davis, L.C. and Verpoorte, R. (2005) Elicitor Signal Transduction Leading to Production of Plant Secondary Metabolites. Biotechnology Advances, 23, 283-333. https://doi.org/10.1016/j.biotechadv.2005.01.003

[11] Shi, L., et al. (2010) Current Progress in the Study on Biosynthesis and Regulation of Ganoderic Acids. Applied Microbiology and Biotechnology, 88, 1243-1251. https://doi.org/10.1007/s00253-010-2871-1

[12] Xu, J.-W., Zhao, W. and Zhong, J.-J. (2010) Biotechnological Production and Application of Ganoderic Acids. Applied Microbiology and Biotechnology, 87, 457-466. https://doi.org/10.1007/s00253-010-2576-5

[13] Zhong, J.-J. and Tang, Y.-J. (2004) Submerged Cultivation of Medicinal Mushrooms for Production of Valuable Bioactive Metabolites. In: Biomanufacturing, Springer, Berlin, 25-59. https://doi.org/10.1007/b94367

[14] Hajjaj, H., et al. (2005) Effect of 26-Oxygenosterols from Ganoderma lucidum and Their Activity as Cholesterol Synthesis Inhibitors. Applied and Environmental Microbiology, 71, 3653-3658. https://doi.org/10.1128/AEM.71.7.3653-3658.2005

[15] Jiang, J., et al. (2008) Ganoderic Acids Suppress Growth and Invasive Behavior of Breast Cancer Cells by Modulating AP-1 and NF- $\kappa$ B Signaling. International Journal of Oncology, 21, 577-584. https://doi.org/10.3892/ijmm.21.5.577

[16] Yue, Q.-X., et al. (2008) Proteomics Characterization of the Cytotoxicity Mechanism of Ganoderic acid D and Computer-Automated Estimation of the Possible Drug Target Network. Molecular \& Cellular Proteomics, 7, 949-961. https://doi.org/10.1074/mcp.M700259-MCP200

[17] Hsu, C.-L., Yu, Y.-S. and Yen, G.-C. (2008) Lucidenic Acid B Induces Apoptosis in Human Leukemia Cells via a Mitochondria-Mediated Pathway. Journal of Agricultural and Food Chemistry, 56, 3973-3980. https://doi.org/10.1021/jf800006u 
[18] Li, F., et al. (2012) Ganoderic Acids Suppress Growth and Angiogenesis by Modulating the NF- $\kappa$ B Signaling Pathway in Breast Cancer Cells. International Journal of Clinical Pharmacology and Therapeutics, 50, 712-721. https://doi.org/10.5414/CP201663

[19] Gao, J.J., et al. (2006) In Vivo Antitumor Effects of Bitter Principles from the Antlered form of Fruiting Bodies of Ganoderma lucidum. Journal of Natural Medicines, 60, 42-48. https://doi.org/10.1007/s11418-005-0003-5

[20] Paterson, R.R.M. (2006) Ganoderma: A Therapeutic Fungal Biofactory. Phytochemistry, 67, 1985-2001. https://doi.org/10.1016/j.phytochem.2006.07.004

[21] Toth, J., et al. (1983) Cytotoxic Triterpenes from Ganoderma lucidum (Polyporaceae): Structures of Ganoderic Acids UZ. Journal of Chemical Research. Synopses, No. 12.

[22] Liu, J., et al. (2009) Ganoderic Acid DM: Anti-Androgenic Osteoclastogenesis Inhibitor. Bioorganic \& Medicinal Chemistry Letters, 19, 2154-2157. https://doi.org/10.1016/j.bmcl.2009.02.119

[23] Tang, W., et al. (2006) Ganoderic Acid T from Ganoderma lucidum Mycelia Induces Mitochondria Mediated Apoptosis in Lung Cancer Cells. Life Sciences, 80, 205-211. https://doi.org/10.1016/j.lfs.2006.09.001

[24] Chen, N.-H., Liu, J.-W. and Zhong, J.-J. (2008) Ganoderic Acid Me Inhibits Tumor Invasion through Down-Regulating Matrix Metalloproteinases 2/9 Gene Expression. Journal of Pharmacological Sciences, 108, 212-216. https://doi.org/10.1254/jphs.SC0080019

[25] Jiang, J., Jedinak, A. and Sliva, D. (2011) Ganodermanontriol (GDNT) Exerts Its Effect on Growth and Invasiveness of Breast Cancer Cells through the Down-Regulation of CDC20 and uPA. Biochemical and Biophysical Research Communications, 415, 325-329. https://doi.org/10.1016/j.bbrc.2011.10.055

[26] Paydary, K., et al. (2013) The Emergence of Drug Resistant HIV Variants and Novel Anti-Retroviral Therapy. Asian Pacific Journal of Tropical Biomedicine, 3, 515-522. https://doi.org/10.1016/S2221-1691(13)60106-9

[27] Min, B.-S., et al. (1998) Triterpenes from the Spores of Ganoderma lucidum and Their Inhibitory Activity against HIV-1 Protease. Chemical and Pharmaceutical Bulletin, 46, 1607-1612. https://doi.org/10.1248/cpb.46.1607

[28] El-Mekkawy, S., et al. (1998) Anti-HIV-1 and Anti-HIV-1-Protease Substances from Ganoderma lucidum. Phytochemistry, 49, 1651-1657. https://doi.org/10.1016/S0031-9422(98)00254-4

[29] Kang, D., Mutakin, M. and Levita, J. (2015) Computational Study of Triterpenoids of Ganoderma lucidum with Aspartic Protease Enzymes for Discovering HIV-1 and Plasmepsin Inhibitors. International Journal of Chemistry, 7, 62. https://doi.org/10.5539/ijc.v7n1p62

[30] Zhang, X.-Q., et al. (2011) Triterpenoids with Neurotrophic Activity from Ganoderma lucidum. Natural Product Research, 25, 1607-1613. https://doi.org/10.1080/14786419.2010.496367

[31] Xia, J., et al. (2018) Epidemiological Characteristics of Malaria from Control to Elimination in Hubei Province, China, 2005-2016. Malaria Journal, 17, 81. https://doi.org/10.1186/s12936-018-2207-7

[32] Dondorp, A.M., et al. (2009) Artemisinin Resistance in Plasmodium falciparum Malaria. New England Journal of Medicine, 361, 455-467. https://doi.org/10.1056/NEJMoa0808859 
[33] Lakornwong, W., et al. (2014) Triterpene Lactones from Cultures of Ganoderma sp. KM01. Journal of Natural Products, 77, 1545-1553. https://doi.org/10.1021/np400846k

[34] Hirotani, M., et al. (1986) Ganoderic Acids T, S and R, New Triterpenoids from the Cultured Mycelia of Ganoderma lucidum. Chemical and Pharmaceutical Bulletin, 34, 2282-2285. https://doi.org/10.1248/cpb.34.2282

[35] Kabir, Y., Kimura, S. and Tamura, T. (1988) Dietary Effect of Ganoderma lucidum Mushroom on Blood Pressure and Lipid Levels in Spontaneously Hypertensive Rats (SHR). Journal of Nutritional Science and Vitaminology, 34, 433-438. https://doi.org/10.3177/jnsv.34.433

[36] Wang, C.-N., et al. (1989) The Aggregation of Human Platelet Induced by Ganodermic Acid S. Biochimica et Biophysica Acta (BBA)-Biomembranes, 986, 151-160. https://doi.org/10.1016/0005-2736(89)90285-X

[37] Shiao, M.-S., et al. (1989) Structures, Biosynthesis and Biological Functions of Oxygenated Treterpenoids in Ganoderma lucidum. Phytochemical Ecology, 9, 235-243.

[38] Yeh, S.-F. (1989) Biosynthesis of Oxygenated Triterpenoids in Ganoderma lucidum. Proceedings of the National Science Council (B).

[39] Hirotani, M., Asaka, I. and Furuya, T. (1990) Investigation of the Biosynthesis of $3 \alpha$-Hydroxy Triterpenoids, Ganoderic Acids T and S, by Application of a Feeding Experiment Using [1, 2-13C2] Acetate. Journal of the Chemical Society, Perkin Transactions, 10, 2751-2754. https://doi.org/10.1039/P19900002751

[40] Hirotani, M. and Furuya, T. (1990) Changes of the Triterpenoid Patterns during Formation of the Fruit Body in Ganoderma lucidum. Phytochemistry, 29, 3767-3771. https://doi.org/10.1016/0031-9422(90)85328-D

[41] Shiao, M.S. (1992) Triterpenoid Natural Products in the Fungus Ganoderma lucidum. Journal of the Chinese Chemical Society, 39, 669-674. https://doi.org/10.1002/jccs.199200102

[42] Abe, I., Rohmer, M. and Prestwich, G.D. (1993) Enzymatic Cyclization of Squalene and Oxidosqualene to Sterols and Triterpenes. Chemical Reviews, 93, 2189-2206. https://doi.org/10.1021/cr00022a009

[43] Bishop, K.S., et al. (2015) From 2000 Years of Ganoderma lucidum to Recent Developments in Nutraceuticals. Phytochemistry, 114, 56-65. https://doi.org/10.1016/j.phytochem.2015.02.015

[44] Chen, S., et al. (2012) Genome Sequence of the Model Medicinal Mushroom Ganoderma lucidum. Nature Communications, 3, 913. https://doi.org/10.1038/ncomms1923

[45] Xu, J.-W., Xu, Y.-N. and Zhong, J.-J. (2012) Enhancement of Ganoderic Acid Accumulation by Overexpression of an N-Terminally Truncated 3-Hydroxy-3-Methylglutaryl Coenzyme A Reductase Gene in the Basidiomycete Ganoderma lucidum. Applied and Environmental Microbiology, 78, 7968-7976. https://doi.org/10.1128/AEM.01263-12

[46] Zhang, W.-X. and Zhong, J.-J. (2010) Effect of Oxygen Concentration in Gas Phase on Sporulation and Individual Ganoderic Acids Accumulation in Liquid Static Culture of Ganoderma lucidum. Journal of Bioscience and Bioengineering, 109, 37-40. https://doi.org/10.1016/j.jbiosc.2009.06.024

[47] Feng, J., et al. (2017) A Novel Ganoderma lucidum G0119 Fermentation Strategy for Enhanced Triterpenes Production by Statistical Process Optimization and Addition of Oleic Acid. Engineering in Life Sciences, 17, 430-439.

https://doi.org/10.1002/elsc.201600071 
[48] You, B.-J., et al. (2013) A Novel Approach to Enhancing Ganoderic Acid Production by Ganoderma lucidum Using Apoptosis Induction. PLoS ONE, 8, e53616. https://doi.org/10.1371/journal.pone.0053616

[49] Xu, Y.-N. and Zhong, J.-J. (2012) Impacts of Calcium Signal Transduction on the Fermentation Production of Antitumor Ganoderic Acids by Medicinal Mushroom Ganoderma lucidum. Biotechnology Advances, 30, 1301-1308.

https://doi.org/10.1016/j.biotechadv.2011.10.001

[50] Li, H.-J., et al. (2016) Further Improvement in Ganoderic Acid Production in Static Liquid Culture of Ganoderma lucidum by Integrating Nitrogen Limitation and Calcium Ion Addition. Bioprocess and Biosystems Engineering, 39, 75-80. https://doi.org/10.1007/s00449-015-1491-7

[51] Zhu, L.-W., Zhong, J.-J. and Tang, Y.-J. (2010) Multi-Fed Batch Culture Integrated with Three-Stage Light Irradiation and Multiple Additions of Copper Ions for the Hyperproduction of Ganoderic Acid and Ganoderma Polysaccharides by the Medicinal Mushroom Ganoderma lucidum. Process Biochemistry, 45, 1904-1911. https://doi.org/10.1016/j.procbio.2010.03.010

[52] Ramirez-Estrada, K., et al. (2016) Elicitation, an Effective Strategy for the Biotechnological Production of Bioactive High-Added Value Compounds in Plant Cell Factories. Molecules, 21, 182. https://doi.org/10.3390/molecules21020182

[53] Ren, A., et al. (2010) Methyl Jasmonate Induces Ganoderic Acid Biosynthesis in the Basidiomycetous Fungus Ganoderma lucidum. Bioresource Technology, 101, 6785-6790. https://doi.org/10.1016/j.biortech.2010.03.118

[54] Giri, C.C. and Zaheer, M. (2016) Chemical Elicitors versus Secondary Metabolite Production in Vitro Using Plant Cell, Tissue and Organ Cultures: Recent Trends and a Sky Eye View Appraisal. Plant Cell, Tissue and Organ Culture, 126, 1-18. https://doi.org/10.1007/s11240-016-0985-6

[55] Zhang, G., et al. (2017) Ethylene Promotes Mycelial Growth and Ganoderic Acid Biosynthesis in Ganoderma lucidum. Biotechnology Letters, 39, 269-275. https://doi.org/10.1007/s10529-016-2238-5

[56] Cao, P.-F., et al. (2017) Effects of Exogenous Salicylic Acid on Ganoderic acid Biosynthesis and the Expression of Key Genes in the Ganoderic Acid Biosynthesis Pathway in the Lingzhi or Reishi Medicinal Mushroom, Ganoderma lucidum (Agaricomycetes). International Journal of Medicinal Mushrooms, 19, 65-73. https://doi.org/10.1615/IntJMedMushrooms.v19.i1.70

[57] Zhai, X., et al. (2017) The Regulatory Mechanism of Fungal Elicitor-Induced Secondary Metabolite Biosynthesis in Medical Plants. Critical Reviews in Microbiology, 43, 238-261. https://doi.org/10.1080/1040841X.2016.1201041

[58] Liu, H., Weng, L. and Yang, C. (2017) A Review on Nanomaterial-Based Electrochemical Sensors for $\mathrm{H}_{2} \mathrm{O}_{2}, \mathrm{H}_{2} \mathrm{~S}$ and NO Inside Cells or Released by Cells. Microchimica Acta, 184, 1267-1283. https://doi.org/10.1007/s00604-017-2179-2

[59] Rőszer, T. (2012) The Biology of Subcellular Nitric Oxide. Springer Science \& Business Media, New York. https://doi.org/10.1007/978-94-007-2819-6

[60] Chen, C., et al. (2017) Transcriptional Profiling Provides New Insights into the Role of Nitric Oxide in Enhancing Ganoderma oregonense Resistance to Heat Stress. Scientific Reports, 7, 15694. https://doi.org/10.1038/s41598-017-15340-6

[61] Gu, L., et al. (2017) Triterpenoid Biosynthesis and the Transcriptional Response Elicited by Nitric Oxide in Submerged Fermenting Ganoderma lucidum. Process Biochemistry, 60, 19-26. https://doi.org/10.1016/j.procbio.2017.05.029 